\title{
Use of clarithromycin and roxithromycin and risk of cardiac death: cohort study
}

\author{
(c) $\frac{1}{10(8)}$ OPEN ACCESS
}

Henrik Svanström statistician, Björn Pasternak postdoctoral fellow, Anders Hviid senior investigator

Department of Epidemiology Research, Statens Serum Institut, 2300 Copenhagen S, Denmark

\author{
Abstract \\ Objective To assess the risk of cardiac death associated with the use \\ of clarithromycin and roxithromycin. \\ Design Cohort study. \\ Setting Denmark, 1997-2011. \\ Participants Danish adults, 40-74 years of age, who received seven \\ day treatment courses with clarithromycin $(n=160297)$, roxithromycin \\ ( $n=588$ 988), and penicillin $V(n=4355$ 309).
}

Main outcome measures The main outcome was risk of cardiac death associated with clarithromycin and roxithromycin, compared with penicillin V. Subgroup analyses were conducted according to sex, age, risk score, and concomitant use of drugs that inhibit the cytochrome P450 3A enzyme, which metabolises macrolides.

Results A total of 285 cardiac deaths were observed. Compared with use of penicillin $\mathrm{V}$ (incidence rate 2.5 per 1000 person years), use of clarithromycin was associated with a significantly increased risk of cardiac death (5.3 per 1000 person years; adjusted rate ratio $1.76,95 \%$ confidence interval 1.08 to 2.85 ) but use of roxithromycin was not (2.5 per 1000 person years; adjusted rate ratio $1.04,0.72$ to 1.51 ). The association with clarithromycin was most pronounced among women (adjusted rate ratios 2.83 (1.50 to 5.36) in women and 1.09 (0.51 to 2.35) in men). Compared with penicillin $\mathrm{V}$, the adjusted absolute risk difference was 37 (95\% confidence interval 4 to 90 ) cardiac deaths per 1 million courses with clarithromycin and 2 (-14 to 25) cardiac deaths per 1 million courses with roxithromycin

Conclusions This large cohort study found a significantly increased risk of cardiac death associated with clarithromycin. No increased risk was seen with roxithromycin. Given the widespread use of clarithromycin, these findings call for confirmation in independent populations.

\section{Introduction}

Acute cardiac toxicity is an increasingly recognised potential adverse effect of antimicrobial drug treatment. ${ }^{1-3}$ Several agents of the macrolide class of antibiotics are known to interfere with the delayed rectifier potassium current $\left(\mathrm{I}_{\mathrm{Kr}}\right)$, which results in accumulation of potassium ions in cardiac myocytes and thereby delays cardiac repolarisation. ${ }^{134}$ Evident on the common electrocardiogram as a prolongation of the QT interval, this mechanism is thought to underlie an increase in the risk of torsade de pointes, a potentially fatal arrhythmia, with macrolides. ${ }^{134}$

Although numerous case reports and spontaneous reports support this notion, ${ }^{15-13}$ evidence from controlled studies to confirm and quantify a potential increase in serious cardiac events risk is limited. Two cohort studies of US Medicaid beneficiaries have found increased risks of sudden cardiac death and cardiovascular death associated with erythromycin and azithromycin, respectively. ${ }^{14}{ }^{15}$ Both studies reported increases in risk in periods of current use of these antibiotics, supporting an acute mechanism. Whereas these studies were performed in cohorts at relatively high baseline cardiovascular risk, a recent population based cohort study of young and middle aged adults at low baseline risk did not find a significantly increased risk of cardiovascular death associated with azithromycin. ${ }^{16}$ The cardiovascular risks associated with exposure to macrolides other than erythromycin and azithromycin are largely unknown, although a small Dutch case-control study pointed towards the possibility of an association between sudden cardiac death and clarithromycin. ${ }^{17}$ Like erythromycin, clarithromycin is metabolised by the cytochrome P450 3A enzyme, thus introducing the potential for a pharmacological interaction with other drugs interfering with this enzyme. ${ }^{1318}{ }^{19}$ Roxithromycin is similarly metabolised by the cytochrome P450 3A enzyme, ${ }^{18} 19$ but it is known to be less susceptible to cytochrome P450 3A inhibition. $^{19}$

Given this background, the cardiac safety profiles of individual macrolides need to be elucidated in greater detail to help guide clinical treatment decisions. We did a nationwide registry based cohort study to investigate the risk of cardiac death associated with clarithromycin and roxithromycin, compared with another antibiotic with similar indications and no known cardiac risk, penicillin $\mathrm{V}$. 


\section{Methods}

We did a prospective study in a historical cohort of users of macrolides and penicillin V in the period 1997-2011. The primary study outcome was cardiac death associated with the use of clarithromycin and roxithromycin, compared with penicillin V. Apart from erythromycin and azithromycin, clarithromycin and roxithromycin are the only macrolides available in Denmark. In subgroup analyses, we additionally assessed the risk of cardiac death according to sex, age, an empirically derived risk score for cardiac death, and concomitant use of cytochrome P450 3A inhibiting drugs.

We took several measures to minimise the potential for confounding. Firstly, to reduce the potential for confounding by indication, we analysed the risk of cardiac death associated with the respective macrolides relative to an active comparator; penicillin $\mathrm{V}$ is the most commonly used antibiotic in Denmark with indications similar to clarithromycin and roxithromycin. The indications for clarithromycin, roxithromycin, and penicillin $\mathrm{V}$ overlap with respect to upper and lower respiratory tract infections and skin and soft tissue infections. Both macrolides are additionally used for chlamydia, mycoplasma, and legionella infections; clarithromycin alone is used for the treatment of peptic ulcer. Secondly, to further reduce the potential for confounding and to increase the likelihood of isolating an effect attributable to the previously hypothesised pro-arrhythmic mechanism, we excluded participants with serious disease, who may be at high baseline risk of death from non-cardiac causes. Thirdly, to account for baseline differences in the risk of cardiac death, we adjusted all analyses for propensity scores, incorporating a wide range of potential confounders. Finally, to assess whether the choice of study drug may be associated with health status at baseline, which could bias the analyses of cardiac death, we also analysed the outcome of other non-cardiovascular death. An increased risk in death due to other causes for any of the study drugs would indicate differential health status at baseline.

We defined the study source population on the basis of the Danish Civil Registration System, ${ }^{20}$ including all people aged 40-74 years living in Denmark during the study period. Using the participants' unique civil registration number, we linked individual level information on drug use, causes of death, and potential confounders. We identified use of macrolides and penicillin V from the nationwide Danish National Prescription Registry. ${ }^{21}$ We included information on all filled prescriptions for study antibiotics during the study period. We considered each prescription to be a separate event, and each participant could contribute multiple prescriptions to the study. For inclusion in the study, the person filling the prescription was required not to have been admitted to hospital and not to have had previous antibiotic treatment within 30 days before and including the prescription fill date. To assure adequate capture of covariate information, participants were required to have been continuously registered in Denmark for at least two years before the prescription fill date. Supplementary table A lists exclusion criteria. We identified cardiac deaths from the Danish Register of Causes of Death, ${ }^{22}$ which is based on death certificates and holds information on causes of all deaths occurring in Denmark, coded according to the International Classification of Diseases (supplementary table B). We collected data on potential confounders at the time of starting treatment (demographic characteristics, medical history and healthcare use, and previous use of other selected drugs) from the Civil Registration System, ${ }^{20}$ the Danish National Patient Register, ${ }^{23}$ and the National Prescription Registry, ${ }^{21}$ respectively (details in supplementary table C).

\section{Statistical analysis}

For the primary analysis, follow-up started on the date when the prescription was filled and ended on the date of the participant becoming 75 years of age, loss to follow-up (disappearance, emigration), end of study (31 December 2011), switch to any other antibiotic, hospital admission, 37 days after the date of filling the prescription, or cardiac or non-cardiac death, whichever occurred first. In the analyses of death due to other causes, hospital admission and switch to another antibiotic were not included as censoring criteria. Given that the study drugs are generally administered in seven day treatment regimens, a follow-up of 37 days allowed effects of treatment to be evaluated for time periods up to 30 days after treatment had ended. Using Poisson regression, we estimated rate ratios comparing individual macrolides with penicillin $\mathrm{V}$ during time periods of current use (0-7 days from start of treatment) and past use (8-37 days). An increase in risk that occurred during current use and disappeared during past use would support the hypothesis of a transient toxic effect; conversely, an increased risk in periods of past use would instead support an alternative mechanism or confounding by baseline characteristics. We also estimated the adjusted absolute difference in risk per 1 million treatment courses with clarithromycin and roxithromycin ((adjusted rate ratio-1)×crude rate among users of penicillin $\mathrm{V})$.

To control for confounding, we adjusted the analyses for the propensity score for starting treatment with, respectively, clarithromycin and roxithromycin. ${ }^{24}$ We derived the propensity scores from two separate logistic regression models, including all courses with the respective study drug and penicillin $\mathrm{V}$ and with all potential confounders listed in table $1 \Downarrow$ included as predictors. We then grouped the study participants according to tenths of propensity score distribution, included as adjustment factors in the regressions of the individual macrolides on cardiac death. To account for possible non-independence between multiple courses in the same participants, we used generalised estimating equations for all analyses.

\section{Cardiac risk score}

We estimated the cardiac risk score for subgroup analysis by using a logistic regression model, including as predictors all potential confounders (table $1 \Downarrow$ ) and a set of dummy variables indicating the treatment received. We then calculated the risk score by multiplying the regression coefficients obtained from the logistic regression model by the respective covariate value at the time of starting treatment for all included factors but the treatment dummy variables, which were all set to $0 .{ }^{25}$ This produced a summarising single value risk score for each treatment course included in the study. We then categorised all courses into three strata according to the score's distribution: low (tenths 1-5), medium (6-8), and high (9-10).

\section{Concomitant use of cytochrome P450 3A inhibiting drugs}

For the subgroup analysis according to concomitant use of cytochrome $\mathrm{P} 4503 \mathrm{~A}$ inhibitors, we identified prescriptions for drugs included in the Indiana University School of Medicine cytochrome $\mathrm{P} 450$ drug interaction table and defined as strong or moderate inhibitors of cytochrome P450 3A (supplementary table D) ${ }^{26}$; a strong inhibitor is defined as a drug that causes a greater than fivefold increase in the area under the plasma 
concentration curve of the substrate or a greater than $80 \%$ decrease in clearance and a moderate inhibitor as a drug that causes a greater than twofold increase in area under the curve or a $50-80 \%$ decrease in clearance. We defined concomitant cytochrome $\mathrm{P} 4503 \mathrm{~A}$ inhibitor use as a prescription that overlapped with the start of antibiotic treatment and the duration of cytochrome P450 3A inhibitor use by the number of defined daily doses in the prescription, with 14 days added to account for irregular drug intake habits.

We considered rate ratios to be statistically significant if the $95 \%$ interval confidence did not overlap 1 . We assessed subgroup homogeneity by using the generalised score statistic. We used SAS 9.4 software for all analyses.

\section{Results}

From a source population of 3379788 people, we identified 8 910459 treatment courses with the study antibiotics. After application of the exclusion criteria, the final study cohort included a total of 5104594 courses: 588988 courses of roxithromycin, 160297 courses of clarithromycin, and 4355 309 courses of penicillin V (fig $1 \Downarrow$ ).

Table $1 \Downarrow$ shows participants' characteristics at baseline. Supplementary table E shows standardised differences between participants with use of the different study drugs. Compared with users of penicillin $\mathrm{V}$, users of clarithromycin and roxithromycin were less likely to be male, were on average slightly older, were less likely to have started treatment in June-August and more likely to have started in December-February, were more likely to have a history of respiratory disease, were more likely to have used drugs for the treatment of asthma and chronic obstructive pulmonary disease, were more likely to have used proton pump inhibitors and $\mathrm{H}_{2}$ blockers, had used a higher number of prescription drugs, were more likely to have had an outpatient hospital contact for non-cardiovascular causes in the previous six months, and were less likely to have had a recent emergency visit. Comparing users of the two macrolides, the groups were well balanced on medical history and use of healthcare; users of clarithromycin were more likely to have started treatment in the early part of the study period and were more likely to have used proton pump inhibitors and $\mathrm{H}_{2}$ blockers. Odds ratio estimates for all potential confounders included in the propensity score models and the cardiac risk score model are shown in tables F and G.

Table $2 \Downarrow$ shows the risk of cardiac death associated with current and past use of clarithromycin and roxithromycin, compared with penicillin V. During follow-up, the proportions censored owing to, respectively, switch to another antibiotic and hospital admission were $19 \%$ and $3 \%$ in courses with clarithromycin, $18 \%$ and $3 \%$ in courses with roxithromycin, and $18 \%$ and $2 \%$ in courses with penicillin V. Among a total of 285 cardiac deaths observed during current use of the study drugs, 18 occurred during use of clarithromycin (incidence rate 5.3 per 1000 person years), 32 during use of roxithromycin ( 2.5 per 1000 person years), and 235 during use of penicillin V (2.5 per 1000 person years). In unadjusted analysis, current use of clarithromycin was associated with a significantly increased risk of cardiac death (rate ratio $2.07,95 \%$ confidence interval 1.28 to 3.35 ); this association persisted after propensity score adjustment (1.76, 1.08 to 2.85). The adjusted absolute risk difference for current use of clarithromycin, compared with use of penicillin $\mathrm{V}$, was 37 (95\% confidence interval 4 to 90 ) cardiac deaths per 1 million treatment courses (fig $2 \Downarrow$ ). With regard to past use, clarithromycin was not associated with an increased risk of cardiac death. Current use of roxithromycin was not associated with an increased risk of cardiac death in adjusted analysis (rate ratio $1.04,0.72$ to 1.51 ). Compared with penicillin $\mathrm{V}$, the adjusted absolute risk difference for current use of roxithromycin was 2 ( -14 to 25 ) cardiac deaths per 1 million courses (fig $2 \Downarrow$ ). We observed no increased risk of cardiac death with past use of roxithromycin in either unadjusted or adjusted analysis. In the analyses of mortality due to other causes, we observed no significantly increased risk with clarithromycin (adjusted rate ratio $0.71,0.49$ to 1.03$)$ or roxithromycin $(1.14,0.95$ to 1.36$)$.

Figure $3 \Downarrow$ shows the risk of cardiac death associated with the use of clarithromycin and roxithromycin in subgroups according to sex, age, cardiac risk score, and concomitant use of cytochrome P450 3A inhibiting drugs. For clarithromycin, the relative risk was higher in women than in men (adjusted rate 1.09 ( 0.51 to 2.35 ) in men and 2.83 (1.50 to 5.36) in women), although not significantly so ( $\mathrm{P}$ for homogeneity $=0.07$ ). We observed no significant differences across subgroups according to age (adjusted rate ratio 2.04 ( 0.88 to 4.74$)$ among participants aged 40-64 years and 1.61 ( 0.89 to 2.91 ) among those aged $\geq 65$ years; $\mathrm{P}=0.67$ ) or concomitant use of cytochrome $\mathrm{P} 4503 \mathrm{~A}$ inhibitors (adjusted rate ratio 1.76 (1.05 to 2.93) without concomitant use of cytochrome $\mathrm{P} 4503 \mathrm{~A}$ inhibitors and 1.62 ( 0.38 to 7.00 ) with concomitant use; $\mathrm{P}=0.92$ ). Estimates were similar across levels of cardiac risk score. For roxithromycin, we observed no significant difference in relative risk between men and women (adjusted rate ratio 0.97 (0.59 to 1.59) in men and 1.13 ( 0.63 to 2.00 ) in women; $\mathrm{P}=0.71$ ) or between those younger than 65 years and those aged 65 years or older (adjusted rate ratio 1.64 (0.95 to 2.85) among participants aged 40-64 years and 0.75 ( 0.45 to 1.25 ) among those aged $\geq 65$ years; $\mathrm{P}=0.06)$. We observed no cardiac deaths among users of roxithromycin with concomitant use of cytochrome $\mathrm{P} 4503 \mathrm{~A}$ inhibitors; accordingly, the effect of concomitant use of cytochrome P450 3A inhibitors with roxithromycin could not be estimated. The relative risk was similar across levels of cardiac risk score.

\section{Sensitivity analyses}

In sensitivity analyses, courses with use of clarithromycin and roxithromycin were matched $(1: 2)$ on the propensity score to courses with use of penicillin V. With respect to both clarithromycin and roxithromycin, the resulting matched cohorts were well balanced on all covariates (supplementary tables $\mathrm{H}$ and I). In propensity score matched analysis for cardiac death, the rate ratio associated with current use of clarithromycin was 1.63 ( 0.87 to 3.03$)$ and the rate ratio associated with current use of roxithromycin was 0.92 (0.60 to 1.39 ).

We also analysed the risk of cardiac death associated with clarithromycin and roxithromycin according to the number of previous courses included in the study for each participant. The distribution for the number of previous courses according to study drug is shown in supplementary table J. For clarithromycin, the rate ratio was 2.07 (1.00 to 4.27) in people who had no previous courses and 0.94 ( 0.49 to 1.82$)$ in those who had one or more previous courses. For roxithromycin, the rate ratio was 0.87 (0.44 to 1.73 ) in people who had no previous courses and 0.84 ( 0.52 to 1.35$)$ in those with previous courses included.

\section{Discussion}

In this study, we investigated the risk of cardiac death associated with the use of clarithromycin and roxithromycin, compared with penicillin $\mathrm{V}$. Use of clarithromycin was associated with a significant $76 \%$ higher risk of cardiac death. We observed no 
significant increase in risk with roxithromycin. In terms of absolute risk, use of clarithromycin would account for an estimated 37 excess cardiac deaths per 1 million courses. In subgroup analyses, the increase in risk with clarithromycin seemed to be driven by a strong association among women.

\section{Comparison with previous studies}

Only limited data exist on the risk of cardiac death associated with clarithromycin and roxithromycin. To our knowledge, no controlled observational studies have been published on the association between roxithromycin and cardiac death. In a Dutch case-control study, use of clarithromycin was found to be associated with a non-significant increased risk of sudden cardiac death, although this analysis was based on only three clarithromycin exposed cases. ${ }^{17}$ In an observational study among Tennessee Medicaid beneficiaries in the United States, the macrolide erythromycin was associated with a significant twofold increased risk of sudden cardiac death. ${ }^{14}$ As in the Medicaid study, the association between clarithromycin use and cardiac death in our study was present with current use of the drug and disappeared in periods of past use. If the association is true, this observation supports an acute toxic mechanism.

In the Medicaid study, a greater than fivefold increase in risk was observed in erythromycin users with concomitant use of cytochrome P450 3A inhibiting drugs. Similar to erythromycin, clarithromycin is metabolised by the cytochrome P450 3A enzyme. ${ }^{14}$ In our study, we did not find an increase in risk among users of clarithromycin with concomitant use of cytochrome P450 3A inhibiting drugs; this analysis, however, was limited by a small number of cases given the uncommon use of cytochrome P450 3A inhibitors. We were unable to examine the possibility that the risk of cardiac death might be increased in users of roxithromycin with concomitant use of cytochrome P450 3A inhibitors. Consequently, the possibility of an increase in risk with concomitant use of cytochrome P450 3A inhibiting drugs (as observed in the study of erythromycin ${ }^{14}$ ) may not be excluded and should be considered in future studies.

Of note, the results suggested that the overall increase in risk with clarithromycin was largely driven by a strong association in women, although the difference in risk compared with men narrowly failed to reach statistical significance. This finding is consistent with female sex being a known risk factor for drug induced cardiac arrhythmia in general and macrolide induced arrhythmia in particular. ${ }^{132728}$ In the previous studies that found increased risk of sudden cardiac death and cardiovascular death with macrolides, subgroup analyses by sex were not reported. ${ }^{14} 15$ In our subgroup analysis by predicted risk score, the rates of cardiac death increased considerably with increasing risk score. No indication of a differential risk according to levels of baseline risk was, however, observed.

In sensitivity analysis, we observed an increase in risk with clarithromycin only in people with no previous antibiotic courses during the study period. This increased risk in first time users could point towards the possibility of a genetic predisposition, which has previously been suggested to underlie drug induced torsade de pointes. ${ }^{3}$ Prolongation of the QT interval and subsequent serious arrhythmia are thought to be the underlying mechanisms for acute cardiac toxicity with macrolides. Individual macrolides vary in their potency of $\mathrm{I}_{\mathrm{Kr}}$ inhibition and thus potential for QT prolongation and, therefore, potentially have different pro-arrhythmic properties. ${ }^{29}$ Notably, available mechanistic data comparing individual macrolides suggest that clarithromycin has higher potency of $\mathrm{I}_{\mathrm{Kr}}$ inhibition compared with roxithromycin. ${ }^{3031}$ If true, the observed increased risk with clarithromycin but not roxithromycin may represent a clinical manifestation of these differing pharmacodynamic properties. Our study expands on the available knowledge of the cardiac safety of macrolides, being the first large scale population based observational study to show significantly increased cardiac risk with clarithromycin and the relative cardiac safety of roxithromycin.

\section{Strengths and weaknesses of study}

Our study has several strengths that merit attention. Firstly, owing to the nationwide coverage of the study, the results are probably widely generalisable. Secondly, the comparative design allowed us to assess roxithromycin and clarithromycin relative to an antibiotic with similar indications, thereby reducing the potential for confounding by indication. Thirdly, to increase the probability of isolating an effect attributable to roxithromycin and clarithromycin, we restricted the study population to people free from serious disease, with no recent use of antibiotics, and no recent hospital admission.

The study also has some limitations. We lacked information on several important lifestyle and health factors that are known to influence the risk of cardiac death, such as smoking and body mass index. Thus, despite propensity score adjustment, residual confounding cannot be ruled out. In propensity score matched analysis, which might provide more robust control for confounders than adjustment, the results, although less precise, were similar to the main findings. Furthermore, the fact that the risk of cardiac death was similar between penicillin $\mathrm{V}$ and both clarithromycin and roxithromycin in periods of past use would indicate that baseline differences between the groups are unlikely to have influenced the results significantly; whereas the incidence of cardiac death during current use of antibiotics is influenced by acute infection (for which the antibiotic was prescribed), the incidence during past use probably resembles the study participants' baseline risk of cardiac death. The fact that no significant differences were observed for mortality due to other causes should further lessen concerns about systematic differences in health status at baseline as an explanation for the observed association; the relatively low risk of death from other causes associated with clarithromycin may indicate that the observed association for cardiac death might be stronger than what was estimated. Data on specific infections for which the drugs were prescribed were not available for this study; however, the fact that clarithromycin and roxithromycin have essentially identical indications should reduce concerns about the results being influenced through an effect of infection rather than the prescribed treatment (that is, confounding by indication). The absence of an association between roxithromycin exposure and cardiac death can be viewed as a negative control for the positive association between clarithromycin and cardiac death.

Furthermore, the primary outcome definition of cardiac death may not have been sufficiently specific to fully capture an increased risk attributable to the postulated pro-arrhythmic effect; any resulting bias would be in the direction of the null and would not explain the association found with clarithromycin. Finally, the rate of cardiac death in this study was low and results were, accordingly, based on few events. The power to detect differences in subgroup analysis may have been limited.

\section{Implications of findings}

In absolute terms, 37 (95\% confidence interval 4 to 90) excess cardiac deaths occurred per 1 million treatment courses associated with current use of clarithromycin compared with current penicillin $\mathrm{V}$ use in this study. Interpretation of the clinical importance of this finding, if confirmed, is delicate. 
One the one hand, the absolute risk is small, so this finding should probably have limited, if any, effect on prescribing practice in individual patients (with the possible exception of patients who have strong risk factors for drug induced arrhythmia). On the other hand, clarithromycin is one of the more commonly used antibiotics in many countries and many millions of people are prescribed this drug each year; thus, the total number of excess (potentially avoidable) cardiac deaths may not be negligible. These factors need to be considered when assessing the overall benefit/risk profile of macrolides (clarithromycin specifically), an important area for future work by, for example, regulatory agencies and other public health officials.

\section{Conclusion}

This nationwide cohort study found a significantly increased risk of cardiac death associated with current use of clarithromycin but not roxithromycin. The observed association for clarithromycin seemed to be largely attributable to women. Before these results are used to guide clinical decision making, confirmation in independent populations is an urgent priority given the widespread use of macrolide antibiotics.

Contributors: All authors contributed to conception and design of the study and to the analysis and interpretation of the study results. HS acquired the data and conducted the statistical analyses. HS and BP drafted the manuscript. All authors critically revised the manuscript and approved the final version for submission. AH supervised the study and is the guarantor.

\section{Funding: None.}

Competing interests: All authors have completed the ICMJE uniform disclosure form at www.icmje.org/coi_disclosure.pdf (available on request from the corresponding author) and declare: no support from any organisation for the submitted work; no financial relationships with any organisations that might have an interest in the submitted work in the previous three years; no other relationships or activities that could appear to have influenced the submitted work.

Ethical approval: The study was approved by the Danish Data Protection Agency. Ethics approval is not required for register based research in Denmark.

Transparency declaration: AH affirms that this manuscript is an honest, accurate, and transparent account of the study being reported; that no important aspects of the study have been omitted; and that any discrepancies from the study as planned have been explained. Data sharing: No additional data available.

1 Justo D, Zeltser D. Torsades de pointes induced by antibiotics. Eur J Intern Med 2006;17:254-9.

2 Mosholder AD, Mathew J, Alexander JJ, Smith H, Nambiar S. Cardiovascular risks with azithromycin and other antibacterial drugs. N Engl J Med 2013;368:1665-8.

3 Owens RC Jr, Nolin TD. Antimicrobial-associated QT interval prolongation: pointes of interest. Clin Infect Dis 2006;43:1603-11.

4 Yap YG, Camm AJ. Drug induced QT prolongation and torsades de pointes. Heart 2003;89:1363-72.
5 Hensey C, Keane D. Clarithromycin induced torsade de pointes. Ir J Med Sci 2008; 177:67-8.

6 Huang BH, Wu CH, Hsia CP, Yin CC. Azithromycin-induced torsade de pointes. Pacing Clin Electrophysiol 2007;30:1579-82.

7 Kamochi H, Nii T, Eguchi K, Mori T, Yamamoto A, Shimoda K, et al. Clarithromycin associated with torsades de pointes. Jpn Circ J 1999;63:421-2.

8 Katapadi K, Kostandy G, Katapadi M, Hussain KM, Schifter D. A review of erythromycin-induced malignant tachyarrhythmia—-torsade de pointes: a case report. Angiology 1997;48:821-6.

9 Kezerashvili A, Khattak H, Barsky A, Nazari R, Fisher JD. Azithromycin as a cause of QT-interval prolongation and torsade de pointes in the absence of other known precipitating factors. J Interv Card Electrophysiol 2007;18:243-6.

10 Koh TW. Risk of torsades de pointes from oral erythromycin with concomitant carbimazole (methimazole) administration. Pacing Clin Electrophysiol 2001;24:1575-6.

11 Piquette RK. Torsade de pointes induced by cisapride/clarithromycin interaction. Ann Pharmacother 1999;33:22-6.

12 Promphan W, Khongphatthanayothin A, Horchaiprasit K, Benjacholamas V. Roxithromycin induced torsade de pointes in a patient with complex congenital atrioventricular block. Pacing Clin Electrophysiol 2003;26:1424-6.

13 Shaffer D, Singer S, Korvick J, Honig P. Concomitant risk factors in reports of torsades de pointes associated with macrolide use: review of the United States Food and Drug Administration Adverse Event Reporting System. Clin Infect Dis 2002;35:197-200.

14 Ray WA, Murray KT, Meredith S, Narasimhulu SS, Hall K, Stein CM. Oral erythromycin and the risk of sudden death from cardiac causes. N Engl J Med 2004;351:1089-96.

15 Ray WA, Murray KT, Hall K, Arbogast PG, Stein CM. Azithromycin and the risk of cardiovascular death. N Engl J Med 2012;366:1881-90.

16 Svanstrom H, Pasternak B, Hviid A. Use of azithromycin and death from cardiovascular causes. N Engl J Med 2013;368:1704-12.

17 Straus SM, Sturkenboom MC, Bleumink GS, Dieleman JP, van der Lei J, de Graeff PA, et al. Non-cardiac QTc-prolonging drugs and the risk of sudden cardiac death. Eur Heart J 2005;26:2007-12.

18 Rubinstein E. Comparative safety of the different macrolides. Int J Antimicrob Agents 2001;18:S71-6.

19 Yamazaki H, Shimada T. Comparative studies of in vitro inhibition of cytochrome P450 3A4-dependent testosterone 6beta-hydroxylation by roxithromycin and its metabolites, troleandomycin, and erythromycin. Drug Metab Dispos 1998;26:1053-7.

20 Pedersen CB. The Danish civil registration system. Scand J Public Health 2011;39(7 suppl):22-5.

21 Kildemoes HW, Sorensen HT, Hallas J. The Danish national prescription registry. Scand $J$ Public Health 2011;39(7 suppl):38-41.

22 Helweg-Larsen K. The Danish register of causes of death. Scand J Public Health 2011;39(7 suppl):26-9.

23 Lynge E, Sandegaard JL, Rebolj M. The Danish national patient register. Scand J Public Health 2011:39(7 suppl):30-3.

24 Brookhart MA, Wyss R, Layton JB, Sturmer T. Propensity score methods for confounding control in nonexperimental research. Circ Cardiovasc Qual Outcomes 2013;6:604-11.

25 Sturmer T, Schneeweiss S, Brookhart MA, Rothman KJ, Avorn J, Glynn RJ. Analytic strategies to adjust confounding using exposure propensity scores and disease risk scores: nonsteroidal antiinflammatory drugs and short-term mortality in the elderly. Am J Epidemio 2005;161:891-8.

26 Indiana University School of Medicine. P450 drug interaction table: Abbreviated "clinically relevant" table. 2013. http://medicine.iupui.edu/clinpharm/ddis/clinical-table/.

27 Kannankeril P, Roden DM, Darbar D. Drug-induced long QT syndrome. Pharmacol Rev 2010;62:760-81.

28 Roden DM. Drug-induced prolongation of the QT interval. N Engl J Med 2004;350:1013-22 .

29 Owens RC Jr. QT prolongation with antimicrobial agents: understanding the significance. Drugs 2004:64:1091-124.

30 Ohtani H, Taninaka C, Hanada E, Otaki H, Sato H, Sawada Y, et al. Comparative pharmacodynamic analysis of Q-T interval prolongation induced by the macrolides clarithromycin, roxithromycin, and azithromycin in rats. Antimicrob Agents Chemother 2000;44:2630-7.

31 Volberg WA, Koci BJ, Su W, Lin J, Zhou J. Blockade of human cardiac potassium channel human ether-a-go-go-related gene (HERG) by macrolide antibiotics. J Pharmacol Exp Ther 2002;302:320-7.

Accepted: 21 July 2014

\section{Cite this as: BMJ 2014;349:g4930}

This is an Open Access article distributed in accordance with the Creative Commons Attribution Non Commercial (CC BY-NC 3.0) license, which permits others to distribute, remix, adapt, build upon this work non-commercially, and license their derivative works on different terms, provided the original work is properly cited and the use is non-commercial. See: http://creativecommons.org/licenses/by-nc/3.0/. 


\section{What is already known on this topic}

Macrolide antibiotics prolong the QT interval and are therefore thought to increase the risk of potentially fatal arrhythmias

\section{What this study adds}

This register based cohort study found a significantly increased risk of cardiac death associated with current use of clarithromycin, which was most pronounced among women

No increased risk of cardiac death was found with roxithromycin

\section{Tables}

\begin{tabular}{|c|c|c|c|}
\hline Characteristics & Roxithromycin ( $n=588$ 988) & Clarithromycin ( $n=160297)$ & Penicillin V ( $n=4355$ 309) \\
\hline No of patients & 350575 & 108767 & 1519324 \\
\hline Male sex & $208343(35.4)$ & $58608(36.6)$ & $1813475(41.6)$ \\
\hline Mean (SD) age, years & $56.6(9.7)$ & $57.2(9.6)$ & $55.7(9.5)$ \\
\hline \multicolumn{4}{|l|}{ Age group, years: } \\
\hline $40-44$ & $88353(15.0)$ & $21506(13.4)$ & $734320(16.9)$ \\
\hline $45-49$ & $86122(14.6)$ & $22078(13.8)$ & $680620(15.6)$ \\
\hline $50-54$ & $90525(15.4)$ & $24833(15.5)$ & $720663(16.5)$ \\
\hline $55-59$ & $95888(16.3)$ & $26947(16.8)$ & 723566 (16.6) \\
\hline $60-64$ & $91146(15.5)$ & $25386(15.8)$ & $625358(14.4)$ \\
\hline $65-69$ & $75181(12.8)$ & $21512(13.4)$ & 485806 (11.2) \\
\hline $70-74$ & $61773(10.5)$ & $18035(11.3)$ & $384976(8.8)$ \\
\hline \multicolumn{4}{|l|}{ Place of birth*: } \\
\hline Denmark & $547639(93.0)$ & $148842(92.9)$ & $4049249(93.0)$ \\
\hline Rest of Europe & $14855(2.5)$ & $4127(2.6)$ & $102731(2.4)$ \\
\hline Outside Europe & $26494(4.5)$ & $7328(4.6)$ & $203329(4.7)$ \\
\hline \multicolumn{4}{|l|}{ Calendar year: } \\
\hline $1997-2001$ & $106094(18.0)$ & $53559(33.4)$ & $1387050(31.8)$ \\
\hline $2002-06$ & $189820(32.2)$ & $54146(33.8)$ & $1486866(34.1)$ \\
\hline $2007-11$ & $293074(49.8)$ & $52592(32.8)$ & $1481393(34.0)$ \\
\hline \multicolumn{4}{|l|}{ Season: } \\
\hline March-May & $142047(24.1)$ & $40446(25.2)$ & $1107762(25.4)$ \\
\hline June-August & $100223(17.0)$ & $26194(16.3)$ & $937113(21.5)$ \\
\hline September-November & $150821(25.6)$ & $39991(24.9)$ & $1053135(24.2)$ \\
\hline December-February & $195897(33.3)$ & $53666(33.5)$ & $1257299(28.9)$ \\
\hline \multicolumn{4}{|l|}{ Medical history: } \\
\hline Acute coronary syndrome & $24156(4.1)$ & $6739(4.2)$ & $163197(3.7)$ \\
\hline Other ischaemic heart disease & $49934(8.5)$ & $13629(8.5)$ & $307176(7.1)$ \\
\hline Heart failure/cardiomyopathy & $13178(2.2)$ & $3772(2.4)$ & $79434(1.8)$ \\
\hline Valve disorders & $5047(0.9)$ & $1262(0.8)$ & $32987(0.8)$ \\
\hline Cerebrovascular disease & $20403(3.5)$ & $5367(3.3)$ & $135715(3.1)$ \\
\hline Arterial disease & $18310(3.1)$ & $5167(3.2)$ & $113997(2.6)$ \\
\hline Arrhythmia & $29728(5.0)$ & $7625(4.8)$ & $181694(4.2)$ \\
\hline Renal disease & $5515(0.9)$ & $1419(0.9)$ & $32776(0.8)$ \\
\hline Serious respiratory disease & $6919(1.2)$ & $2449(1.5)$ & $29406(0.7)$ \\
\hline Other respiratory disease & $78485(13.3)$ & $24642(15.4)$ & $335736(7.7)$ \\
\hline Psychiatric disorder & $24346(4.1)$ & $6254(3.9)$ & $141921(3.3)$ \\
\hline Cardiac surgery in previous year & $2098(0.4)$ & $656(0.4)$ & $17584(0.4)$ \\
\hline
\end{tabular}


Table 1 (continued)

Characteristics

Prescription drug use in previous year:

\begin{tabular}{|c|c|c|c|}
\hline ARB/ACE-I & $105302(17.9)$ & $25884(16.1)$ & $642512(14.8)$ \\
\hline Dihydropyridine CCB & $50266(8.5)$ & $12022(7.5)$ & $317213(7.3)$ \\
\hline Non-dihydropyridine CCB & $12236(2.1)$ & $3787(2.4)$ & $80466(1.8)$ \\
\hline Loop diuretics & $39307(6.7)$ & $11835(7.4)$ & $227858(5.2)$ \\
\hline Other diuretics & $90091(15.3)$ & $23503(14.7)$ & $572287(13.1)$ \\
\hline$\beta$ blockers & $65721(11.2)$ & $16661(10.4)$ & $446204(10.2)$ \\
\hline Anti-arrhythmics class I and III & $2159(0.4)$ & $636(0.4)$ & $14857(0.3)$ \\
\hline Digoxin & $6704(1.1)$ & $2019(1.3)$ & $48771(1.1)$ \\
\hline Nitrates & $16894(2.9)$ & $5093(3.2)$ & $107981(2.5)$ \\
\hline Platelet inhibitors & $74129(12.6)$ & $18861(11.8)$ & $459602(10.6)$ \\
\hline Anticoagulants & $11655(2.0)$ & $2754(1.7)$ & $81964(1.9)$ \\
\hline Lipid lowering drugs & $87153(14.8)$ & $19087(11.9)$ & $495742(11.4)$ \\
\hline Oral anti-diabetes drugs & $27058(4.6)$ & $6282(3.9)$ & $174266(4.0)$ \\
\hline Insulin & $11999(2.0)$ & $2936(1.8)$ & $83053(1.9)$ \\
\hline Antidepressants & $81350(13.8)$ & $21213(13.2)$ & $510268(11.7)$ \\
\hline Antipsychotics & $18141(3.1)$ & $4974(3.1)$ & $138339(3.2)$ \\
\hline Anxiolytics, hypnotics, and sedatives & $125545(21.3)$ & $38501(24.0)$ & $844880(19.4)$ \\
\hline$\beta_{2}$ agonist inhalants & $92409(15.7)$ & $29464(18.4)$ & $434233(10.0)$ \\
\hline Corticosteroid inhalants & $101348(17.2)$ & $32736(20.4)$ & $441846(10.1)$ \\
\hline Xantines & $8248(1.4)$ & $3537(2.2)$ & $37856(0.9)$ \\
\hline Anticholinergic inhalants & $20875(3.5)$ & $6490(4.0)$ & $67306(1.5)$ \\
\hline Oral corticosteroids & $61815(10.5)$ & $19598(12.2)$ & $305743(7.0)$ \\
\hline NSAIDs & $196256(33.3)$ & $51520(32.1)$ & $1414481(32.5)$ \\
\hline Opiates & $83453(14.2)$ & $23073(14.4)$ & $521681(12.0)$ \\
\hline Systemic hormone replacement therapy & $79431(13.5)$ & $25331(15.8)$ & $519201(11.9)$ \\
\hline Anti-osteoporotic drugs & $13403(2.3)$ & $3874(2.4)$ & $63089(1.4)$ \\
\hline $\mathrm{PPI} / \mathrm{H}_{2}$ blocker & $104313(17.7)$ & $35083(21.9)$ & $592932(13.6)$ \\
\hline \multicolumn{4}{|l|}{ Vo of prescription drugs used in previous year: } \\
\hline $1-5$ & $323735(55.0)$ & $83350(52.0)$ & $2871871(65.9)$ \\
\hline $6-9$ & $146273(24.8)$ & $40501(25.3)$ & $921393(21.2)$ \\
\hline$\geq 10$ & $118980(20.2)$ & $36446(22.7)$ & $562045(12.9)$ \\
\hline \multicolumn{4}{|l|}{ Jse of healthcare in previous six months: } \\
\hline Cardiovascular ED visit/hospital admission & $10680(1.8)$ & $3084(1.9)$ & $71770(1.6)$ \\
\hline Other ED visit/hospital admission & $62136(10.5)$ & $17310(10.8)$ & $450149(10.3)$ \\
\hline Cardiovascular outpatient contact & $14350(2.4)$ & $3849(2.4)$ & $95501(2.2)$ \\
\hline Other outpatient contact & $155873(26.5)$ & $40786(25.4)$ & $910646(20.9)$ \\
\hline ED visit in previous 7 days & $2309(0.4)$ & $512(0.3)$ & $49416(1.1)$ \\
\hline
\end{tabular}

ACE-I=angiotensin converting enzyme inhibitor; $A R B=$ angiotensin receptor blocker; $C C B=$ calcium channel blocker; ED=emergency department; NSAID=non-steroidal anti-inflammatory drug; $\mathrm{PPI}=$ proton pump inhibitor.

${ }^{*}$ Missing values on place of birth $(0.5 \%)$ were replaced with mode imputation. 
Table 2| Risk of cardiac death associated with use of clarithromycin and roxithromycin compared with penicillin $\mathbf{V}$

\begin{tabular}{|c|c|c|c|c|}
\hline & \multirow[b]{2}{*}{ Cardiac deaths } & \multirow[b]{2}{*}{ Incidence rate/1000 patient years } & \multicolumn{2}{|c|}{ Rate ratio $(95 \% \mathrm{Cl})$} \\
\hline & & & Unadjusted & Propensity score adjusted \\
\hline \multicolumn{5}{|l|}{ Current use } \\
\hline Clarithromycin & 18 & 5.3 & 2.07 (1.28 to 3.35$)$ & 1.76 (1.08 to 2.85$)$ \\
\hline Roxithromycin & 32 & 2.5 & 1.00 (0.69 to 1.44$)$ & 1.04 (0.72 to 1.51$)$ \\
\hline Penicillin V & 235 & 2.5 & 1.00 (reference) & 1.00 (reference) \\
\hline \multicolumn{5}{|l|}{ Past use } \\
\hline Clarithromycin & 14 & 1.3 & 1.24 (0.73 to 2.13$)$ & 1.06 (0.62 to 1.82$)$ \\
\hline Roxithromycin & 42 & 1.0 & 1.01 (0.73 to 1.40$)$ & $1.06(0.76$ to 1.46$)$ \\
\hline Penicillin V & 308 & 1.0 & 1.00 (reference) & 1.00 (reference) \\
\hline
\end{tabular}




\section{Figures}

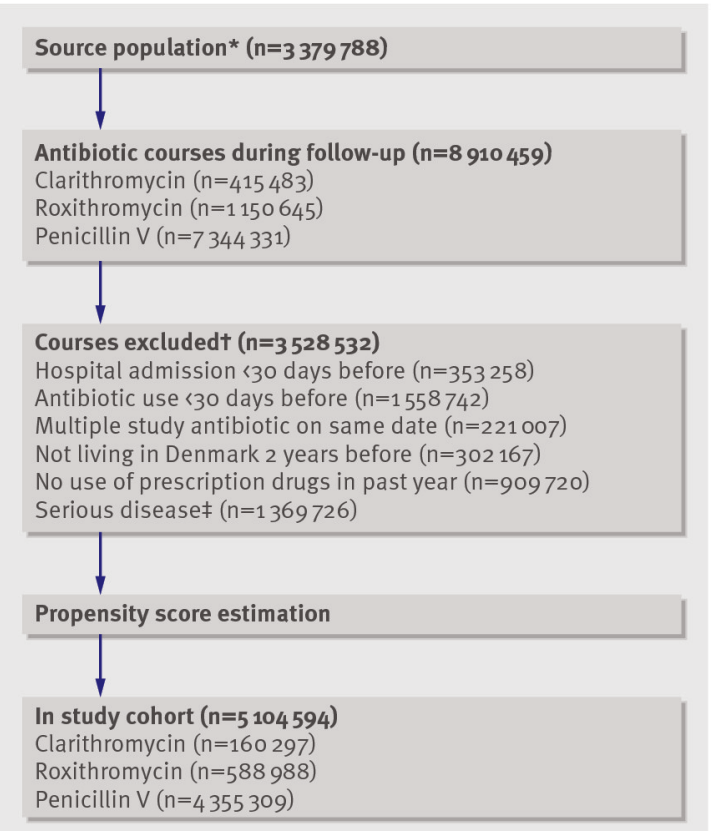

Fig 1 Enrolment of participants in cohort of users of clarithromycin, roxithromycin, and penicillin V. *People living in Denmark, aged 40-74 years, 1997-2011. †Numbers do not sum because some participants were excluded for more than one reason. łIncluding cancer, serious neurological disease, congenital anomalies/childhood conditions, liver disease,

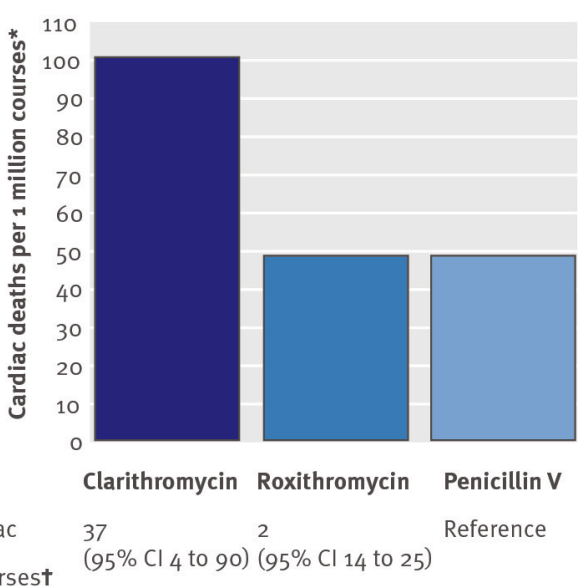

Excess cardiac deaths per $(95 \% \mathrm{Cl} 4$ to 90$)(95 \% \mathrm{Cl} 14$ to 25$)$

Fig 2 Rate of cardiac death and number of excess cardiac deaths with clarithromycin and roxithromycin, compared with penicillin V. *As calculated from unadjusted rate of cardiac death. †Adjusted for propensity scores 


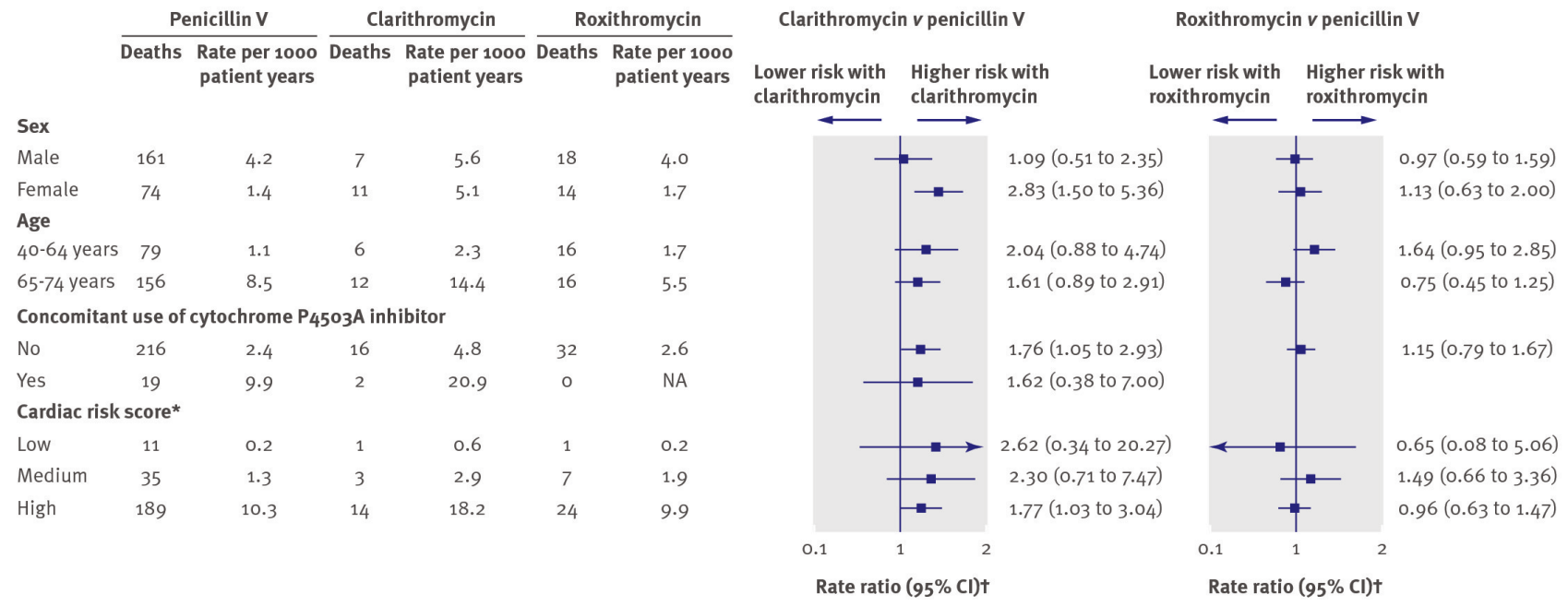

Fig 3 Subgroup analyses of risk of cardiac death associated with clarithromycin and roxithromycin, compared with penicillin V. ${ }^{*}$ Cardiac risk score was covariate summary score aiming to capture baseline risk of cardiac death and derived from all variables shown in table 1; score was categorised according to tenths of score's distribution and strata defined as low (tenths 1-6), medium (6-8), and high (9-10). †Adjusted for propensity score, categorised according to tenths of score's distribution; analyses stratified according to cardiac risk score were not adjusted for propensity scores 